\title{
Development of a Virtual Laboratory for the Study of Mechanics
}

\author{
Masoud Rais-Rohani, Debbie T. Brown \\ Mississippi State University
}

\begin{abstract}
Virtual Laboratory for the Study of Mechanics (VLSM) is a Web-based educational tool being developed to support Statics and Mechanics of Materials instruction at Mississippi State University. It is designed to serve as an asynchronous tutorial tool with ten modules devoted to topics of interest in these two courses. A typical module consists of concise theoretical discussions, interactive MathView ${ }^{\circledR}$ examples, a Java-enabled design modeling and analysis tool, and a collection of test-your-knowledge exercises with response feedback. This paper describes the objectives of developing VLSM, and gives an overview of some of its modules that have already been developed and are currently being used and evaluated at the designated Web site (www.ae.msstate.edu/vlsm).
\end{abstract}

\section{Introduction}

The use of computers in engineering education is not new. According to the U.S. Department of Education, engineering faculty have been the quickest to adopt learning technologies. Since the Fall of 1992, engineering faculty have surpassed all other disciplines by using computational tools and software to implement computer-aided instruction. ${ }^{1}$ A significant shift in technology implementations that is apparent in engineering education today is toward greater use of computers (and other devices) as tools in the learning process rather than as instructional delivery devices. Many of the newer technology tools are designed to facilitate educational tasks by providing students with aids for studying content and practicing skills. Computer-aided instructional reinforcements allow instructors to make more efficient use of limited lecture periods by focusing more on the crucial concepts and less on the mundane tasks.

Computer-based instruction is commonly divided into two modes of delivery: Synchronous and Asynchronous. Synchronous delivery of instruction involves presentation to the student while the instructor is simultaneously available for student-contact with immediate feedback. Examples of synchronous instruction on the computer would be instructor-moderated chat-room discussions, instructor-facilitated application activities, and instructor-driven slide shows, to name a few. Asynchronous delivery of instruction involves self-directed learning on the part of the student with delayed teacher feedback and indirect student contact. Asynchronous instruction examples would include tutorials, bulletin boards, email discussion lists, and automated computer/Web applications that compile student activity data for teacher review at a later time.

The explosive growth of potentially useful content on the World Wide Web, coupled with new, easier-to-use development tools, has encouraged some engineering faculty to explore the use of Web technologies for their course materials. ${ }^{2,3}$ In the past few years there has been a noticeable 
progress toward the use of computer technology in Statics and Mechanics of Materials courses. ${ }^{4-6}$ The two subjects represent fundamental engineering courses in many fields of engineering, and as such demand considerable attention in preparing students for more advanced topics in engineering.

The College of Engineering at Mississippi State University instituted a policy that requires all incoming freshmen in the Fall of 1999 to own their own personal computers. Accompanying this requirement, the college has provided financial support to a select group of faculty to develop additional electronic courseware for discipline-specific as well as more general or fundamental subjects in engineering. The tool described in this paper represents a major effort supported by this computer initiative.

\section{Virtual Laboratory for the Study of Mechanics}

Virtual Laboratory for the Study of Mechanics (VLSM) is a Web-based educational tool being developed primarily to support Statics and Mechanics of Materials courses at Mississippi State University. VLSM is designed to encompass most of the topics studied in these courses, and to serve as an asynchronous tutorial tool. It can be used by the instructor to enhance his lecture presentations and by individual students to improve their understanding of concepts in solid mechanics and their ability to analyze various rigid and elastic structural systems. The objectives of developing VLSM can be summarized as follows:

$>$ Provide students with an online tutorial tool that would

- help enhance their knowledge and understanding of solid mechanics

- present the important concepts in a manner that is easy to comprehend

- $\quad$ provide example problems that can be altered to answer "what if" questions

- allow them to design and solve problems for various response parameters

- present the solution procedure consistent with the methods taught in each course

- $\quad$ answer their frequently asked questions

- provide them with interactive exercises to improve their problem solving skills

- offer them the opportunity to post questions to a specific instructor

$>$ Provide instructors with a tool that would

- help make their lecture presentation more effective

- enable them to experiment with an alternate method of teaching

- reduce the lecture time devoted to mundane aspects of the course

- facilitate teaching students with different learning styles

- assist them in organizing collaborative study sessions

Once fully developed, VLSM will have ten modules as identified in Fig. 1. A typical module consists of concise theoretical discussions, interactive MathView ${ }^{\circledR}$ example problems, a Javaenabled design and analysis tool, and a collection of test-your-knowledge exercises. With one year into the three-year project, three of its modules (i.e., Geometric Properties of Shapes, Characteristics and Properties of Materials, and Trusses) have been mostly completed and are currently being evaluated at the designated Web site (www.ae.msstate.edu/vlsm). 


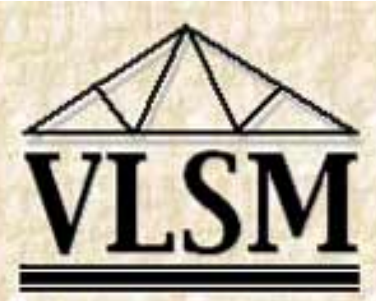

\section{$V_{\text {irtual Laboratory for the }} S_{\text {tudy of }} M_{\text {echanics }}$}

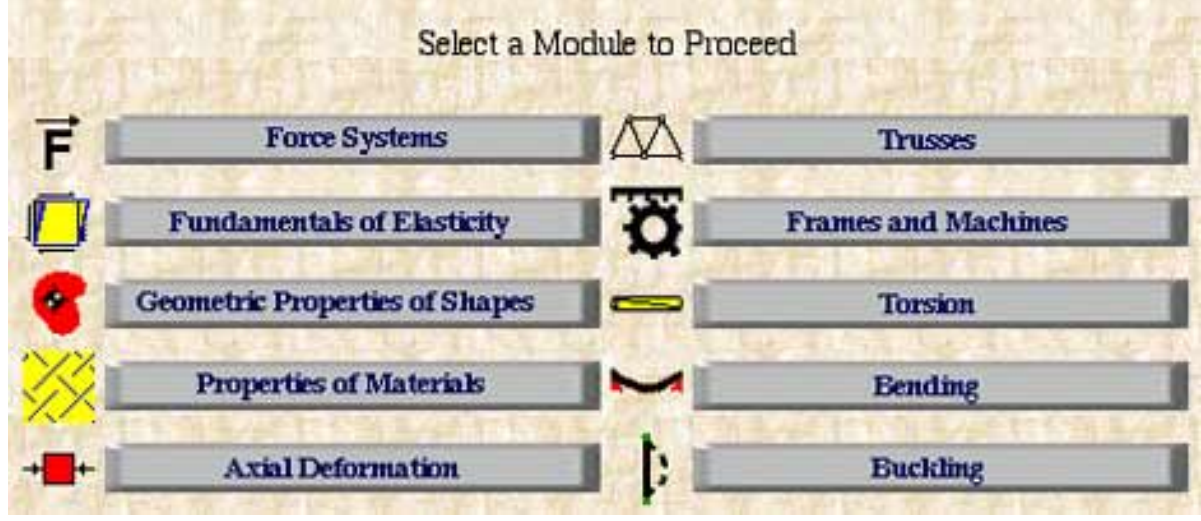

Fig. 1 List of modules in VLSM

\section{Trusses}
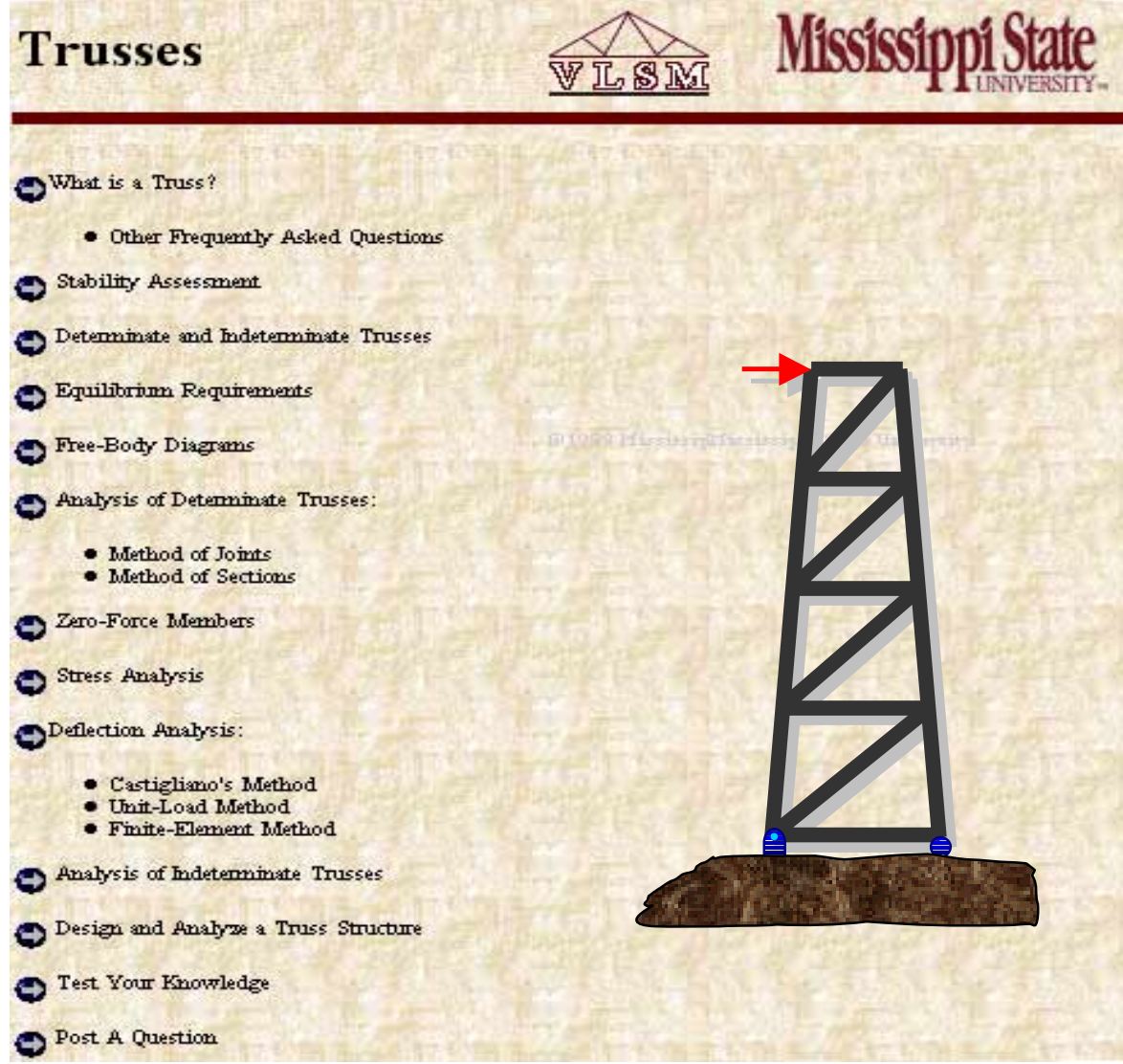

Fig. 2 The index page of the Trusses module 
Upon entering VLSM, one can simply click on the title button to go to the desired module. Figure 2 shows the index page of the Trusses module. A three-frame layout is used with the global navigation buttons located at the top of the page and the local navigation buttons listed vertically at the left side of the screen. A simple point and click action is all that is needed to navigate through the system. The right frame is used to display the contents of each section.

\section{Theoretical Discussions}

Each VLSM module is divided into a number of sections focusing on different aspects of the main theme of the module. A discussion of the underlying theory is presented in a brief and descriptive fashion with the help of mathematical formulae, graphs, and charts. Each discussion tends to provide a more concise version of a typical classroom lecture, and it highlights the important aspects of the theory as well as the specific details on the corresponding methods of analysis.

\section{Pop Question: (Question appears by clicking on the hyperlink)}

Identify the zero-force members in the truss shown below.

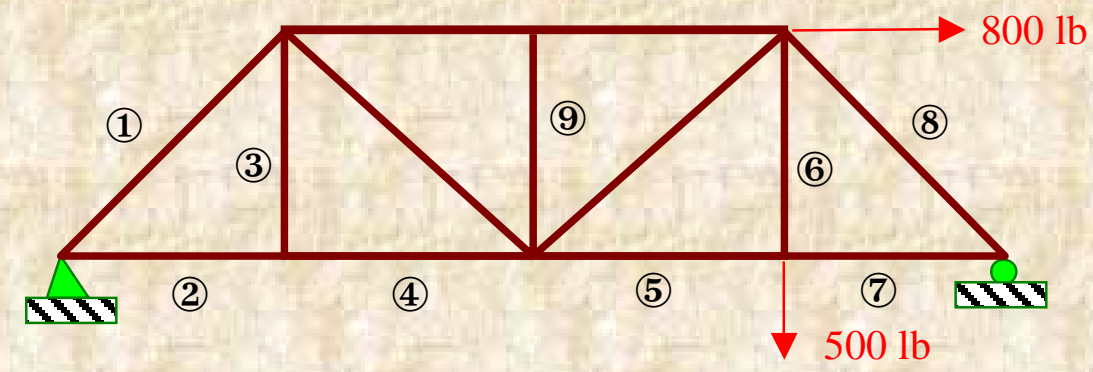

You can submit one answer at a time by leaving the remaining answer fields blank.

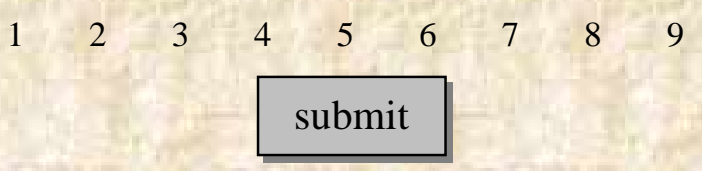

If a wrong member such as number 1 is chosen:

Sorry! Member 1 is not a zero-force member. Think about the definition and try again.

If a right member such as number 3 is chosen:

Good! You found a zero-force member. Can you find another?

If numbers 3 and 9 are chosen:

Good! You got the right answer. Do you know Why the right answer is ...

Looking at the truss, we observe members 3 and 9 have one thing in common. They are both zero-force members. Member 3 is perpendicular to members 2 and 4, and there is no external force or support reaction at the common joint. Therefore, we can conclude that it is a zero-force member. The same analogy applies to member 9 .

Fig. 3 A pop question example in Trusses module 
In an attempt to increase the effectiveness of the tutorial lectures, a number of "Pop Questions" with multiple-choice answers are used as shown in Fig. 3. Each selected answer invokes an automated response, which may also include an additional question. These questions tend to provoke the curiosity of even those students who tend to be too shy to ask questions in class in the company of their classmates. This is one of the features of VLSM that promotes active learning by encouraging students to pay attention to the theoretical discussions that precede each question.

In addition to the pop questions, each module has a section devoted entirely to a collection of frequently asked questions and answers. In the event a student has a question that is not included on this list, he or she can simply post that question to his or her instructor. The VLSM's post-aquestion feature can be used to e-mail a question to a particular instructor who is teaching the section of Statics or Mechanics of Materials to which the student belongs. The posted question and the instructor's response would then be added to the list of frequently asked questions for future reference. The list of instructors is updated at the beginning of each semester to include only the names of those teaching different sections of the two solid mechanics courses.

The theoretical discussions were developed originally using Microsoft Word, and saved as HTML documents with DHTML features and JavaScript codes added in later. The layout of each page was then reorganized using Macromedia’s Dreamweaver $2^{\circledR}$ software.

\section{Interactive Example Problems}

One feature that makes an electronic tutorial tool superior to a printed textbook is the level and scope of interactivity that can be introduced between the user and the tool. This feature has been emphasized in VLSM with the help of interactive example problems. Below the problem statement in each interactive example, a MathView-based solution is shown in a separate window as depicted in Fig. 4.

MathView ${ }^{\circledR}$ is a symbolic mathematical software marketed by Waterloo Maple ${ }^{*}$ for the purpose of providing interactive mathematical solution algorithms on the Web. It also has graphics capability that can be used to generate accurate plots of various parameters in the problem.

Prior to its placement on VLSM, the solution to each example problem is developed in symbolic form using MathView. These solution notebooks are then saved as .thp executable files that can be viewed and manipulated by a Web browser (on Macintosh or Windows operating systems). In these example problems, the user can alter the input data and almost instantaneously see the effects trickle down through the solution sequence including the embedded graphics.

The interactive examples show students how a solution should be formulated and executed, and provide them with means of exploring different scenarios by changing the input data. Through the examination of various "what if" questions, students can develop a deeper understanding of the analysis method and the effect each parameter has on the solution results.

\footnotetext{
* MathView has since been acquired by Theorist Interactive, LLC., and is currently being marketed and supported under the name LiveMath®.
} 
Area Moments of Inertia: Example

If the composite area shown is divided into four individual rectangular pieces, determine the moments of inertia of each piece with respect to its own centroidal horizontal and vertical axes.
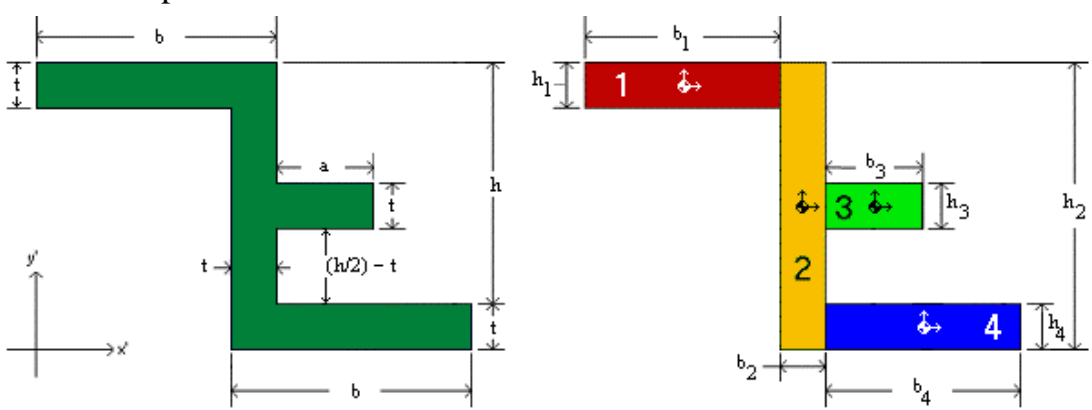

Q Data:

$\square \mathrm{b}=10 \square \mathrm{h}=9 \quad \square \mathrm{t}=1 \quad \square \mathrm{a}=5$

Q Youcan highlight and change one or all of the parameters give n above to see the effects on the solution

\section{QSolution Procedure:}

Since we are asked to solve for the moments of inertia of individual rectangular pieces, the re is no need to know the location of centroid of the composite shape. All we need to know is the centroidal position of each rec tangle as a separate piece. For a rectangle of width $b$ and heighth, the moments of ine rtia about its ce ntroidal horizontal and vertical axes, identified as $x$ and $y$, are givenas

$\square \mathrm{Ixx}=\frac{1}{12} \mathrm{bh}^{\mathrm{J}} \quad \square \mathrm{Iyy}=\frac{1}{12} \mathrm{hb}^{\mathrm{J}}$

$Q$ The width and height dimensions of each rec tangular piece are ide ntified by inspection to be

$\square b_{1}=b-t \quad \square h_{1}=t$

$\square b_{2}=t \quad \square h_{2}=h+t$

$\square b_{3}=a \quad \square h_{3}=t$

$\square b_{4}=b-t \quad \square h_{4}=t$

QSubstituting the proper values into the moments of inertia equations yields

Qpiece 1:

$$
\begin{aligned}
& \square I x_{1}=\frac{1}{12} b_{1} h_{1}{ }^{J} \quad \Delta I x x_{1}=\frac{1}{12} t^{3}(b-t) \Delta I x_{1}=0.75 \\
& \square \text { Iyy }_{1}=\frac{1}{12} \mathrm{~h}_{1} \mathrm{~b}_{1}{ }^{\mathrm{s}} \Delta \mathrm{Iyy}_{1}=\frac{1}{12} \mathrm{t}(\mathrm{b}-\mathrm{t})^{\mathrm{J}} \Delta \mathrm{Iyy}_{1}=60.75 \\
& \mathrm{Q} \\
& \square \operatorname{Ixx}_{2}=\frac{1}{12} \mathrm{~b}_{2} \mathrm{~h}_{2}{ }^{J} \quad \Delta \operatorname{Ixx}_{2}=\frac{1}{12} \mathrm{t}(\mathrm{h}+\mathrm{t})^{\jmath} \quad \Delta \mathrm{Ixx}_{2}=83.333 \\
& \square \text { Iyy }_{2}=\frac{1}{12} h_{2} b_{2}{ }^{J} \quad \Delta \text { Iyy }_{2}=\frac{1}{12} t^{\mathrm{J}}(\mathrm{h}+\mathrm{t}) \quad \Delta \mathrm{Iyy}_{2}=0.83333
\end{aligned}
$$

Q Piece 3:

$$
\begin{aligned}
& \square \mathrm{Ixx}_{\mathrm{J}}=\frac{1}{12} \mathrm{~b}_{\mathrm{J}} \mathrm{h}_{\mathrm{J}} \mathrm{J}_{\mathrm{J}} \quad \Delta \mathrm{Ixx}_{\mathrm{J}}=\frac{1}{12} \mathrm{at}^{\mathrm{J}} \quad \Delta \mathrm{Ixx}_{\mathrm{J}}=0.41667 \\
& \square \mathrm{Iyy}_{\mathrm{J}}=\frac{1}{12} \mathrm{~h}_{\mathrm{J}} \mathrm{b}_{\mathrm{J}}{ }^{\mathrm{S}} \Delta \mathrm{IVy}_{\mathrm{J}}=\frac{1}{12} \mathrm{a}^{\mathrm{J}} \mathrm{t} \quad \Delta \mathrm{Iyy}_{\mathrm{J}}=10.417 \\
& \square \mathrm{Ixx}_{4}=\frac{1}{12} \mathrm{~b}_{4} \mathrm{~h}_{4}^{\mathrm{J}} \quad \Delta \mathrm{Ixx}_{4}=\frac{1}{12} \mathrm{t}^{\mathrm{J}}(\mathrm{b}-\mathrm{t}) \quad \Delta \mathrm{Ixx}_{4}=0.75 \\
& \square \text { Iyy }_{4}=\frac{1}{12} h_{4} b_{4}{ }^{3} \quad \Delta \text { Iyy }_{4}=\frac{1}{12} t(b-t)^{3} \quad \Delta \text { Iyy }_{4}=60.75
\end{aligned}
$$

Q Area Moment of Inertia Declarations

Fig. 4 A MathView example problem in Geometric Properties of Shapes module 


\section{Design and Analysis Tools}

A typical module in VLSM is equipped with a design and analysis tool that allows a student to develop a design model for which it provides a step by step solution. An example of this is the Shape Design and Analysis Tool (S-DAT) in the Geometric Properties of Shapes module. Developed using Sun's JDK 1.2 software, S-DAT can be used to draw a rather complex geometric shape, and to obtain its properties such as centroid, moments of inertia, product of inertia, etc. Figure 5-a shows the graphical user interface (GUI) for S-DAT. The icons on the right show the ten built-in templates. They can be used individually or in combination to generate a more complex shape.

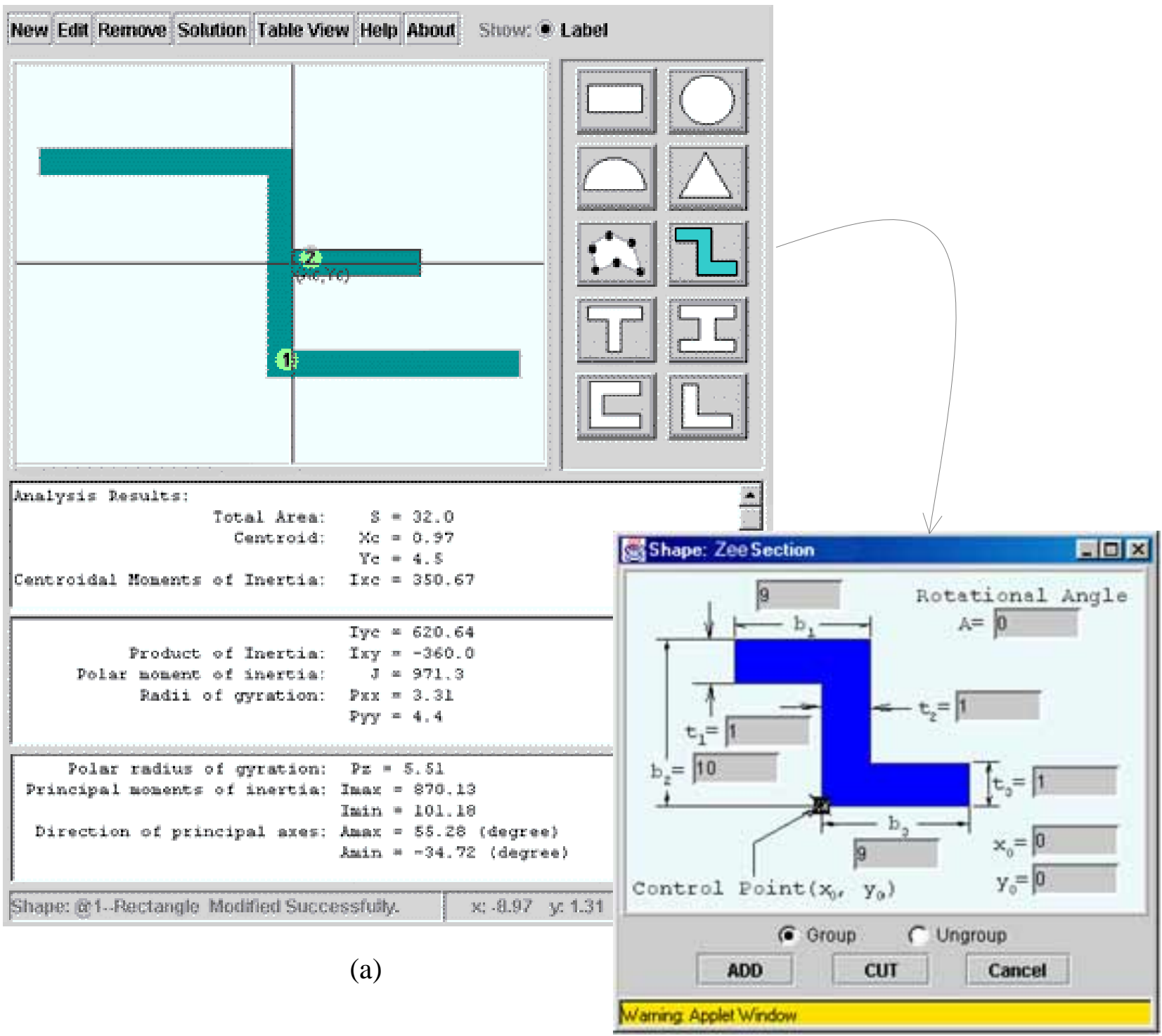

(b)

Fig. 5 Shape Design and Analysis Tool in VLSM, (a) Main GUI, (b) Zee template

The composite shape shown in Fig. 5-a is a combination of a Zee section, identified as element1, and a rectangle, identified as element 2 . Figure 5-b shows the Zee template, which appears by 
clicking on the Zee icon. The desired dimensions and orientation angle can be specified in the designated fields. The control point is used to facilitate the creation of composite shapes by positioning each element in the desired location. If the chosen template represents a hole in the composite shape, then the "CUT" button, as shown in Fig. 5-b, is pressed, otherwise the "ADD" button is used. If the "Ungroup" option is selected, the shape shown in the template will be divided into its basic elements. For example, a Zee section can be ungrouped into three separate rectangles with the user being able to eliminate or modify the size and orientation of each rectangular element.

As soon as a shape appears on the S-DAT window, its geometric properties are immediately calculated with the results displayed at the bottom of the screen (see Fig. 5-a). As more elements are added to or subtracted from the baseline geometry, the solution results are automatically updated. Figure 5-a shows the output screen in three separate scrolled positions. The key feature of S-DAT is that it shows the student the steps leading to each answer. By clicking on the "Solution" icon at the top of the GUI, a separate window appears showing the solution sequence. This feature enables the student to learn what information and formulae are used to calculate each geometric property.

The Truss Design and Analysis Tool (T-DAT) in the Trusses module can be used for dynamic modeling and analysis of various truss structures. It was developed primarily with the Microsoft Visual Studio for J++ and Visual Interdev using JavaScript and VBScript programming languages. T-DAT can be used to develop and analyze any two-dimensional truss structure. On the client side, it is compatible with Netscape version 4.0 or higher, and requires that Java/JavaScript be enabled. T-DAT currently requires a Windows NT Web server running IIS, FrontPage Server Extensions, and ASP Server Extensions, with the SQL Server database engine.

The layout of T-DAT consists of 5 frames as shown in Fig. 6, each having a distinct function in the overall operation of the tool:

1. The Banner frame contains the menu buttons with tool tips, and controls the transitions between design (or modeling) and analysis operational modes.

2. The Comments frame contains user instructions and processing hints related to the current state of the tool. Comment data are retrieved from a state table contained in a SQL Server database using Active Server Page (ASP) technology and VBScript interfaces.

3. The Display frame contains a Java applet called "ModelBuilder" which provides the graphical representation of the truss.

4. The Output frame is used to dynamically display the analysis and solution to the user.

5. The Input frame is used for all user data and analysis interactions.

When operating in the design mode, the student can use T-DAT to define the geometry of any 2dimensional truss structure, as well as the materials, boundary conditions, and external loads. TDAT also has a collection of built-in models that can be used for classroom demonstrations or as practice problems by students. It also allows each model to be modified in terms of joint location, member connectivity, magnitude and location of external forces, and location and form of supports. Hence, it is possible to modify a single problem into several different problems in order to see the effects of various parameters on support reactions and internal member forces. 


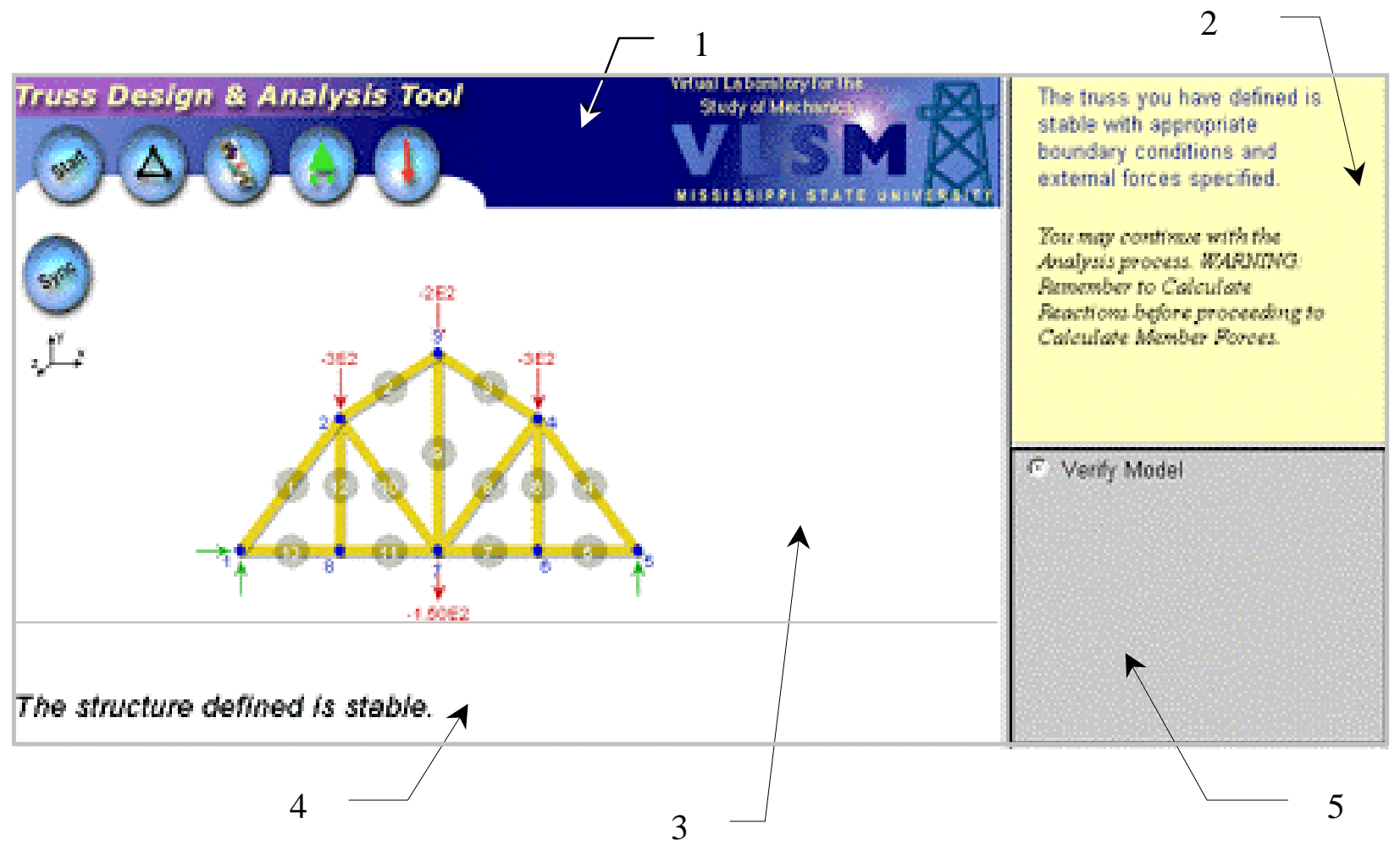

Fig. 6 A truss model developed using T-DAT

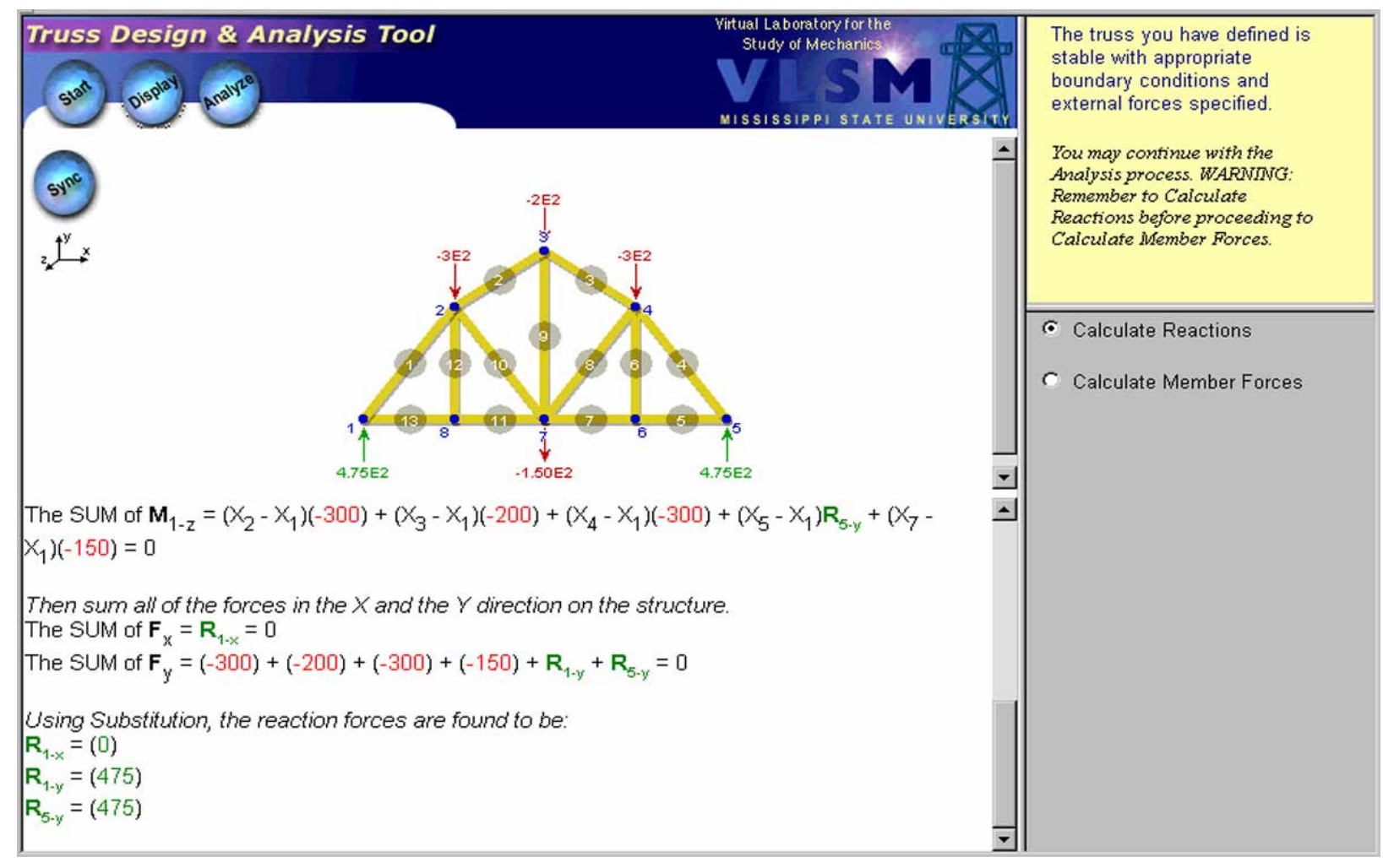

Fig. 7 T-DAT showing the calculation of reaction forces 
Once the model is defined, the student initiates the steps necessary to perform the analysis. The T-DAT analysis engine was designed with the purpose of helping students to solve truss problems using the methods of analysis taught in both elementary and advanced courses in solid mechanics. After verifying the structure for stability, the student would proceed to calculate the reaction forces at the supports. When the student clicks on the "Calculate Reactions" button in the Input frame, T-DAT develops the solution for exhibit in the Output frame as shown in Fig. 7. The applet is updated to show the free-body diagram of the whole truss including the reaction forces.

In the Statics course, students are taught two different methods (i.e., Method of Joints, and Method of Sections) by which the internal forces of a truss may be determined. In T-DAT, the student can specify the solution to the problem based on either one of the two methods. In each case, he or she can see exactly how the solution is formulated, and how the results are obtained. This is perhaps the most notable feature that sets T-DAT apart from other truss analysis tools that simply provide the user with the final answers without demonstrating how those answers are obtained.

To illustrate this feature, the screen shot in Fig. 8 captures a step in the Method of Joints analysis process. The student is asked to select a joint for analysis. The applet then "zooms-in" on the selected joint and updates the diagram with the internal member forces (represented by blue arrows) while the Output frame shows the mathematical equations used to obtain the solution.

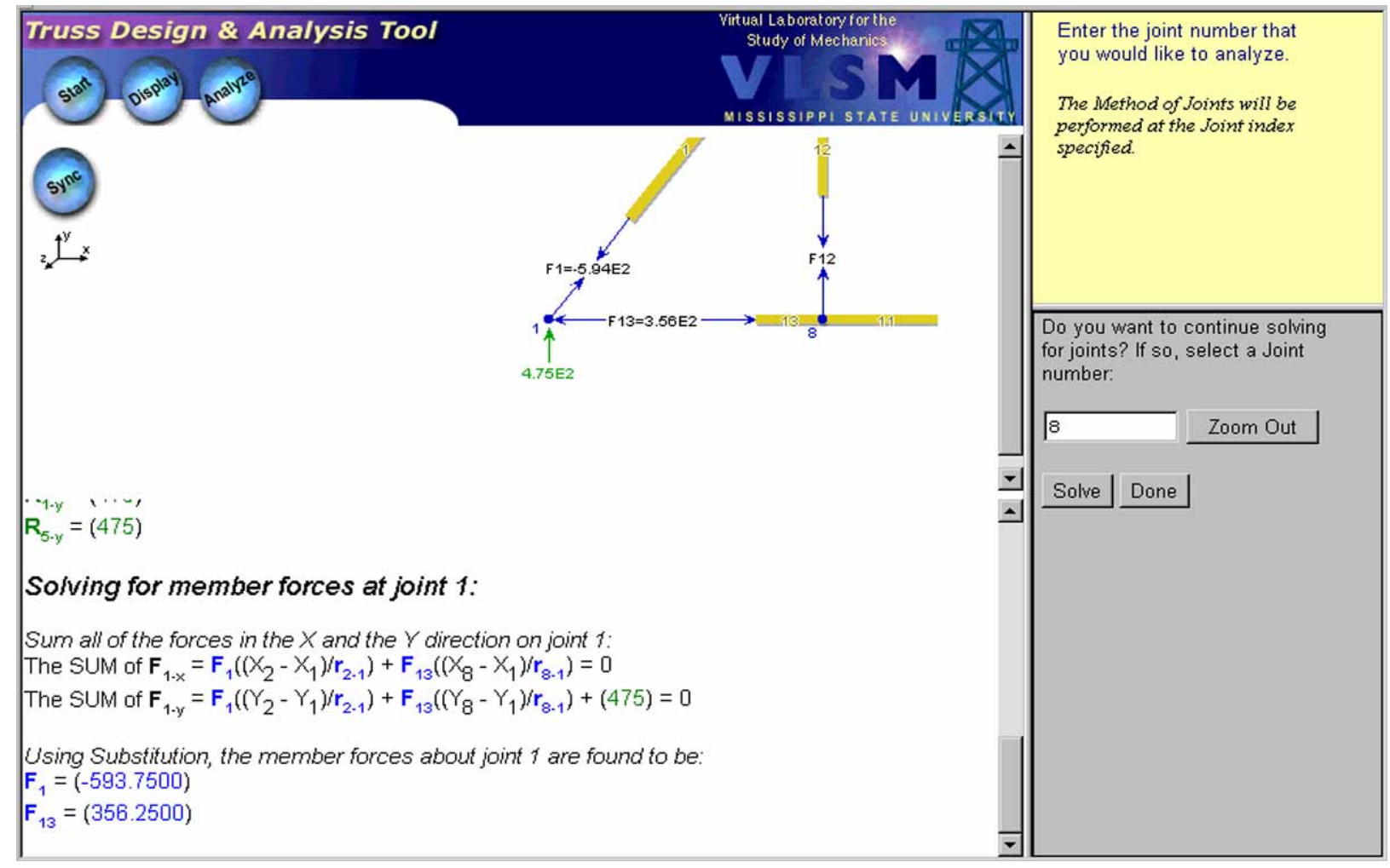

Fig. 8 T-DAT showing the truss analysis using the Method of Joints 
In the Method of Sections analysis process, the user is directed to select up to 3 members to split and cut the truss into two distinct sections; the ModelBuilder displays the line of separation and the section labels. The user is then instructed to choose a section for analysis. Upon this selection, the applet redraws only those joints and members included in the selected section as shown in Fig. 9.

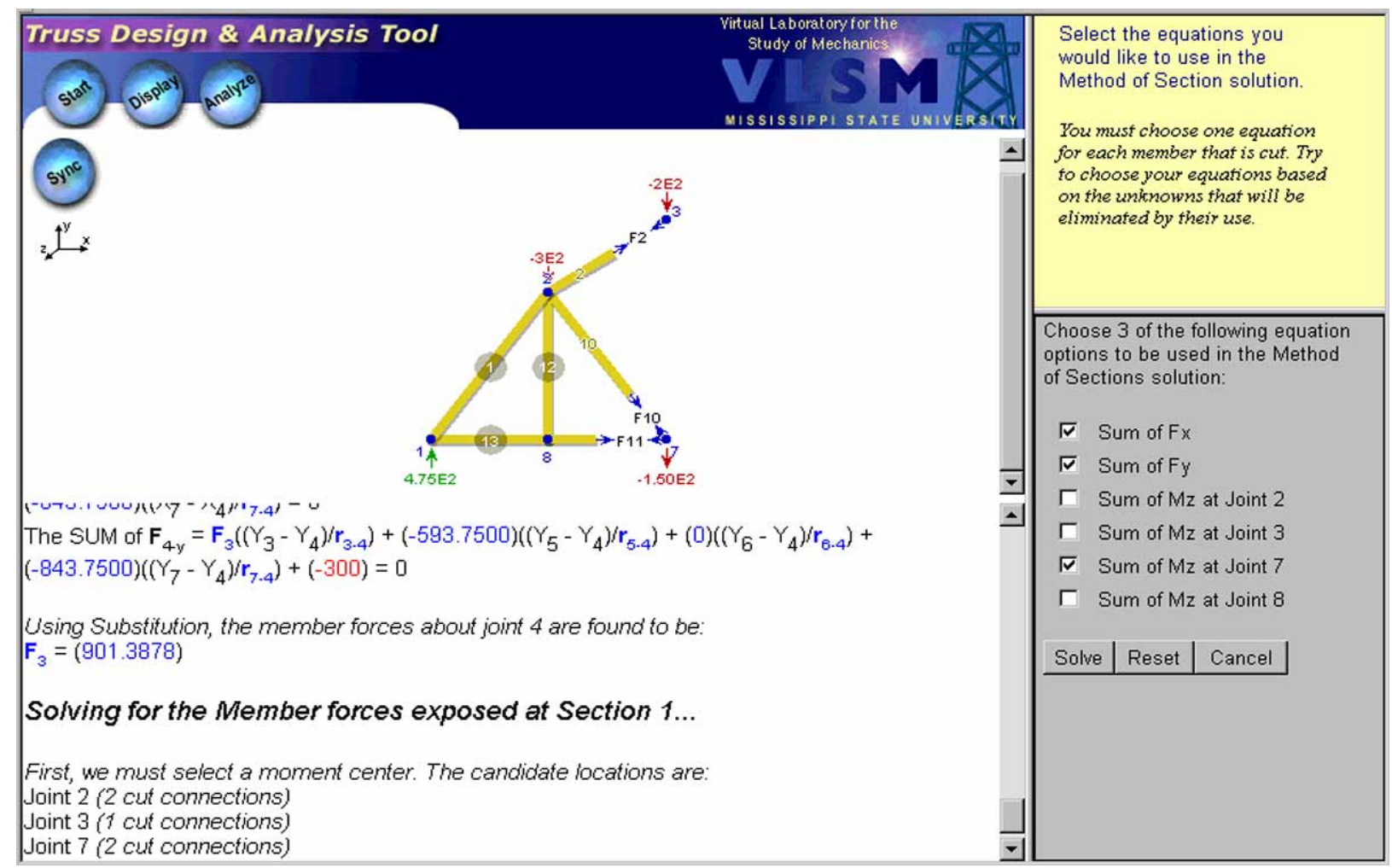

Fig. 9 T-DAT showing the truss analysis using the Method of Sections

The Method of Sections analysis engine then asks the student to select, among the list of options, the appropriate set equations for use in the analysis. By trying different options, the student can learn the most efficient way of analyzing this or other similar truss structures.

Therefore, by demonstrating to the students how the solution is formulated and how the results are checked and validated, T-DAT can help to enhance their ability to work such problems on their own without any outside help, which is our primary goal.

\section{Test-Your-Knowledge Exercises}

Testing has a significant role in education, and in the case of online education, assessment could help to determine how well the student has understood the concepts in solid mechanics and how well he or she can apply the methods of analysis to the solution of a given problem. The testyour-knowledge exercises in each module represent a collection of problems to which the student must supply the answers. The questions range in degree of difficulty. In some exercises the correct answers must be selected from a set of multiple choices while in others the student 
must first perform an analysis and then input the numerical value for the answer sought in the problem.

Figure 10 shows a test-your-knowledge exercise in the Geometric Properties of Shapes module. The response to the correct answer for the first question is shown followed by the sequence of responses for the three unsuccessful attempts to answer the second question.

\section{Exercise 6}

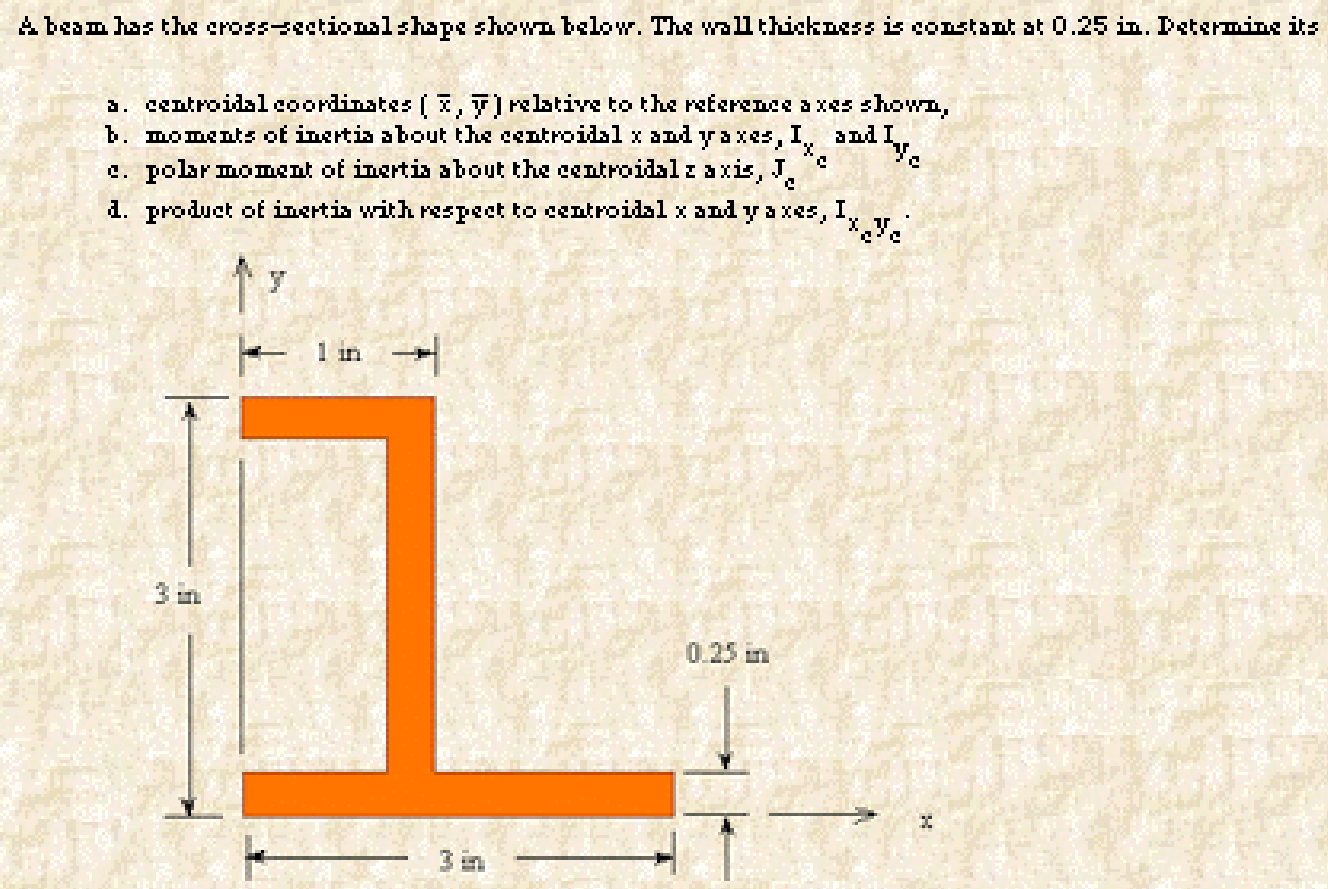

Round up your answers to three decimal places. You ean submit one answer at a time by leaving the remaining answer tields blank or you ean submit all answers together.

$$
\begin{aligned}
& \bar{x}=\sqrt{1.106} \text { in } \bar{y}=\sqrt{1.5} \text { in } I_{x_{c}}=\square \quad \text { in } I_{y_{c}}=\square \quad \text { in }^{4} \\
& J_{c}=\square_{\text {in }^{4}} I_{x_{c} y_{c}}=\square_{\text {in }^{4}} \\
& \text { submit }
\end{aligned}
$$

Good Job! You got the right answer tor $\bar{x}$.

Somy! Your answer for $\overline{\mathrm{y}}$ is incomet. Keep in mind that in $\overline{\mathrm{y}}$ equation $\mathrm{y}_{i}$ is the distance measured trom the reterence $x$ a xis to the centroid of the $i^{\text {th }}$ element. Also check to be sure that the area you are caleulating tor each element is comect. You can caleulate the centroidal location by dividing the composite area into three rectangular elements. Try again or review the section on centroid of area.

Somy! Your answer tor $\bar{y}$ is incorrect. The total ares of the shape is $1.625 \mathrm{in}^{2}$. If your total area is comect, then check the terms involving $y_{i} A_{i}$. Try again or review the section on centroid of area.

Somy! Your answer tor $\overline{\mathrm{y}}$ is incomet. Here is how the centroidal location is caleulated. Review it caretully.

Fig. 10 A test-your-knowledge exercise in Geometric Properties of Shapes module 
The built-in response feature praises the student for finding the right answer, and-more importantly-provides hints when the answer to a question is wrong. Although the system has no restrictions in terms of the number of times the answer to a question is changed by the student, it provides hints only up to the third unsuccessful attempt. The first hint, in response to a wrong answer, is usually general in nature and makes sure that the student is on the right track. The second hint provides a more specific suggestion and may also give the numerical values to certain items that must be calculated prior to finding the final answer. The third and final hint includes a hyperlink to the solution sequence leading to the answer sought. When a problem requires multiple answers, the student can provide one answer at a time or all together. When the solution is shown to the student it involves only the question for which the student-supplied answer is wrong. The hint system uses a set of predetermined responses to each question, and is not based on artificial intelligence. The accuracy of each numerical answer is determined within a set tolerance.

These exercises are not developed for the purpose of assigning a grade to an individual student; rather they are created to provide students with self-evaluation tools that may be used to improve their problem solving skills prior to an actual test given by the course instructor.

Each exercise is made interactive with the use of DHTML and JavaScript programming languages.

\section{Preliminary Assessment and Future Work}

VLSM is currently being evaluated by students and instructors in multiple sections of Statics and Mechanics of Materials courses at Mississippi State University. We plan to improve the quality and efficiency of existing as well as future modules based on the feedback we receive from our constituency. The anecdotal information we have collected so far indicates that the design and analysis tools and the test-your-knowledge exercises are the most liked and utilized features of VLSM.

Despite our goal of developing a Web based tool that would not be platform specific, the current technology has prevented us from achieving this goal. We are particularly disappointed by the inability of Macintosh operating systems to interact with the Java applets in the design and analysis tools in VLSM. We hope this problem will be fixed in the soon-to-be-released Mac OS 10 .

We are currently working on the development of the Torsion module. In addition, we are working on the development of several interactive applets that simulate the physical response of structural members to applied loads. The remaining modules of VLSM are scheduled for completion by summer of 2001 .

Acknowledgment

VLSM is being developed through financial support from the Hearin Foundation. The Learning Technologies Group at the MSU/NSF Engineering Research Center for Computational Field Simulation provided the technical and financial support for the development of T-DAT. The development support provided by the students (Qunying Shi, Shannon Brown, Hemang Maniar, and Michael Hazlewood) is also gratefully acknowledged. 
Bibliography

1. U.S. Department of Education, "Condition of Education 1999, Section III, Quality of Education Environments," April 1999, pages 64-65.

2. Rais-Rohani, M. and Young K.A., "Development of a Multimedia Structural Mechanics Teaching Tool on the World Wide Web," Proceedings of the 1996 ASEE Annual Conference, Washington, D.C., June 23-26, 1996.

3. Rencis, J.J., Flory, E., Kwok, P., and Alam, J., "Learning Modules for Finite Element Method on the World Wide Web," Proceedings of the 1999 ASEE Annual Conference, Charlotte, NC, June 20-23, 1999.

4. Jack, H., "A Paperless (almost) Statics Course," Proceedings of the 1998 ASEE Annual Conference, Seattle, WA, June 21-24, 1998.

5. Li, J. and Lee, M.Y., "Teaching Mechanics with Multimedia Tools," Proceedings of the 1999 ASEE Annual Conference, Charlotte, NC, June 20-23, 1999.

6. Gramoll, K., "Teaching Statics Online with only Electronic Media on Laptop Computers," Proceedings of the 1999 ASEE Annual Conference, Charlotte, NC, June 20-23, 1999.

\section{MASOUD RAIS-ROHANI}

Masoud Rais-Rohani is an Associate Professor of Aerospace Engineering and Engineering Mechanics at Mississippi State University (MSU) where he teaches and conducts research in the area structural mechanics with focus on aircraft structures. He received his Ph.D. in Aerospace Engineering from Virginia Tech in 1991, and is a registered Professional Engineer in the state of Mississippi.

\section{DEBBIE T. BROWN}

Debbie Brown is a research engineer with the Center for Educational and Training Technology at Mississippi State University. She received her BS in Computer Engineering from MSU in 1987, and has since accumulated 12 years of commercial application development and learning technology experience. She is also currently serving as Secretary of the IEEE Learning Technology Standards Committee. 